\title{
Extraplanar Hı regions in the edge-on spiral galaxies NGC 3628 and NGC 4522^
}

\author{
Y. Stein ${ }^{1}$, D. J. Bomans ${ }^{1,2}$, A. M. N. Ferguson ${ }^{3}$, and R.-J. Dettmar ${ }^{1,2}$ \\ 1 Astronomisches Institut (AIRUB), Ruhr-Universität Bochum, Universitätsstrasse 150, 44801 Bochum, Germany \\ e-mail: stein@astro.rub.de \\ 2 Research Department: Plasmas with Complex Interactions, Ruhr-Universität Bochum, Universitätsstrasse 150, 44801 Bochum, \\ Germany \\ 3 Institute for Astronomy, University of Edinburgh, Blackford Hill, Edinburgh EH9 3HJ, UK
}

Received 9 February 2017 / Accepted 30 May 2017

\section{ABSTRACT}

\begin{abstract}
Context. Gas infall and outflow are critical for determining the star formation rate and chemical evolution of galaxies but direct measurements of gas flows are difficult to make. Young massive stars and HII regions in the halos of galaxies are potential tracers for accretion and/or outflows of gas.

Aims. Gas phase abundances of three HII regions in the lower halos of the edge-on galaxies NGC 3628 and NGC 4522 are determined by analyzing optical long-slit spectra. The observed regions have projected distances to the midplane of their host from 1.4 to $3 \mathrm{kpc}$. Methods. With the measured flux densities of the optical nebular emission lines, we derived the oxygen abundance $12+\log (\mathrm{O} / \mathrm{H})$ for the three extraplanar HII regions. The analysis was based on one theoretical and two empirical strong-line calibration methods.

Results. The resulting oxygen abundances of the extraplanar HII regions are comparable to the disk HII regions in one case and are a little lower in the other case. Since our results depend on the accuracy of the metallicity determinations, we critically discuss the difference of the calibration methods we applied and confirm previously noted offsets. From our measurements, we argue that these three extraplanar HII regions were formed in the disk or at least from disk material. We discuss the processes that could transport disk material into the lower halo of these systems and conclude that gravitational interaction with a companion galaxy is most likely for NGC 3628 while ram pressure is favored in the case of NGC 4522.
\end{abstract}

Key words. galaxies: abundances - ISM: abundances - galaxies: individual: NGC 3628 - galaxies: individual: NGC 4522 HII regions - galaxy: evolution

\section{Introduction}

Star formation in spiral galaxies usually takes place in dense regions of the interstellar medium (ISM) close to the midplane of the disk. However, there is evidence for low-level star formation high above the midplane in the Milky Way and in some external galaxies. OB stars exist at large distances from the plane in the Milky Way (e.g., Greenstein \& Sargent 1974; Kilkenny et al. 1995) and in the halos of other galaxies (e.g., Comerón et al. 2001). Furthermore, extraplanar HII regions have been detected in galaxies with a strong indication for an exchange of matter between the disk and the halo; see, for example, NGC 55 (Ferguson et al. 1996), NGC 891, and NGC 4013 (Howk \& Savage 1999), as well as the Virgo galaxy NGC 4402 (Cortese et al. 2004). In some objects, such extraplanar HII regions are found at even larger galactocentric distances and hence are attributed to the intracluster or intergalactic medium of interacting galaxies, where the HII regions probably formed in tidal debris (Mendes de Oliveira et al. 2004; Ryan-Weber et al. 2004), or by ram pressure stripping, such as in the Virgo cluster (Gerhard et al. 2002; Oosterloo \& van Gorkom 2005).

There are different scenarios to explain the presence of young stars and star formation in these unusual environments.

\footnotetext{
* Based on observations gathered as part of observing program 64.N0208(A), 3.6 m telescope with European Southern Observatory (ESO) Faint Object Spectrograph and Camera (EFOSC2) at ESO, La Silla observatory.
}

(I) For the young stars, ejection from the disk by either asymmetric supernovae of a close binary system (e.g., Stone 1982) or dynamical three- or four-body encounters in dense stellar systems (e.g., Poveda et al. 1967) could be responsible for their existence far from the galactic midplane. Supersonic space motions of massive stars are possible with this mechanism, as is apparent from bow shocks induced by these stars (e.g., Gvaramadze \& Bomans 2008). (II) Star formation in the halo itself would not only explain the presence of young stars in the halos (e.g., Keenan et al. 1986) but would also explain the existence of HII regions in the halo, formed with halo material. (III) Tidal forces could induce clumps in material drawn out of galactic disks or in gas falling back onto a disk (e.g., Barnes \& Hernquist 1996), which then would form stars and thus explain extraplanar HII regions.

One way to constrain the origin of the above-mentioned star formation in galactic halos is the determination of metallicities of the extraplanar and disk HII regions. Low metallicities of extraplanar regions in comparison to high metallicity disk regions would indicate that the region is formed by low metallicity halo gas (second scenario). Comparable metallicities between disk and extraplanar regions would lead to the conclusion that the extraplanar HII region is formed by disk material (first and third scenario).

Tüllmann et al. (2003) studied extraplanar HII regions in the galaxy NGC 55. They found a lower metallicity of the extraplanar regions in comparison to HII regions in the midplane 
Table 1. Galaxy parameter and observation log.

\begin{tabular}{|c|c|c|c|c|c|c|c|c|c|c|c|}
\hline Galaxy & $\begin{array}{c}\text { RA (J2000) } \\
{[1]}\end{array}$ & $\begin{array}{c}\operatorname{Dec}(\mathrm{J} 2000) \\
{[2]}\end{array}$ & $\begin{array}{c}\text { Hubble type } \\
{[3]}\end{array}$ & $\begin{array}{c}v\left[\mathrm{~km} \mathrm{~s}^{-1}\right] \\
{[4]}\end{array}$ & $\begin{array}{c}D[\mathrm{Mpc}] \\
{[5]}\end{array}$ & $\begin{array}{c}i\left[^{\circ}\right] \\
{[6]}\end{array}$ & $\begin{array}{c}\text { Grism\# } \\
{[7]}\end{array}$ & $\begin{array}{c}t_{\text {int }}[\mathrm{s}] \\
{[8]}\end{array}$ & $\begin{array}{l}\text { PA } \\
{[9]}\end{array}$ & $\begin{array}{c}\text { Seeing } \\
{[10]}\end{array}$ & $\begin{array}{l}\mathrm{AM} \\
{[11]}\end{array}$ \\
\hline \multirow[t]{2}{*}{ NGC 3628} & $11^{\mathrm{h}} 20^{\mathrm{m}} 17^{\mathrm{s}}$ & $13^{\circ} 35^{\prime} 23^{\prime \prime}$ & $\mathrm{Sb} \mathrm{pec}$ & $843 \pm 1$ & $12.2 \pm 2.4$ & $87^{1}$ & 7 & $2 \times 1800$ & $-15.50^{\circ}$ & 1.11 & 1.40 \\
\hline & & & & & & & 9 & $2 \times 1800$ & & 1.11 & 1.38 \\
\hline \multirow[t]{4}{*}{ NGC 4522} & $12^{\mathrm{h}} 33^{\mathrm{m}} 39.7^{\mathrm{s}}$ & $09^{\circ} 10^{\prime} 30^{\prime \prime}$ & $\mathrm{SB}(\mathrm{s}) \mathrm{cd}$ & $2329 \pm 1$ & $16.8 \pm 1.3$ & $75 \pm 5^{2}$ & $7 \mathrm{~s} 1$ & $1 \times 1200$ & $-70.92^{\circ}$ & n.a. & 1.34 \\
\hline & & & & & & & $9 \mathrm{~s} 1$ & $2 \times 1200$ & & n.a. & 1.34 \\
\hline & & & & & & & $7 \mathrm{~s} 2$ & $1 \times 1200$ & $-70.90^{\circ}$ & n.a. & 1.88 \\
\hline & & & & & & & $9 \mathrm{~s} 2$ & $1 \times 1200$ & & n.a. & 1.71 \\
\hline
\end{tabular}

Notes. Right ascension [1] and declination [2] of the galaxies from NED. [3] Hubble type from de Vaucouleurs et al. (1991a). radial velocity [4], and distance [5] (if available averaged distances are used) were taken from NED. [6] is the inclination, [7] the grism number and the slit number (s1, s2 for NGC 4522), [8] shows the integration time, [9] is the position angle of the slit, [10] is the seeing (not available for NGC 4522), and [11] is the airmass.

References. ${ }^{(1)}$ Tully (1988), ${ }^{(2)}$ Kenney et al. (2004).

and concluded that the second scenario is most likely responsible and that the HII regions formed from halo material. This result was verified by Kudritzki et al. (2016). The metallicities of the extragalactic HII regions in Stephan's Quintet (Mendes de Oliveira et al. 2004), in the interacting galaxies studied by Ryan-Weber et al. (2004), and in the extraplanar HII regions in the Virgo galaxy NGC 4402 are all enhanced in metallicity. In the case of the Virgo galaxy NGC 4388, subsolar metallicities are observed. This suggests there may be no single mechanism for producing extraplanar HII regions. However, there have only been a limited number of studies so far and the nature and origin of such objects requires further study.

NGC 3628 in the Leo Triplet and NGC 4522 in the Virgo Cluster are two edge-on galaxies. These galaxies are known to have extended diffuse $\mathrm{H} \alpha$ structure, which we refer to as extraplanar diffuse ionized gas (eDIG; Rossa \& Dettmar 2000). Three extraplanar HII regions are known in these systems. We here present optical long-slit spectroscopy of these regions, which we analyze to determine metallicities and thus place further constraints on the origins of the young stars and gas at large scale heights.

The extraplanar HII region (1) of NGC 4522 is offset from the main star-forming region defined by $\mathrm{H} \alpha$ and lies at a projected distance of $1.4 \mathrm{kpc}$. This is also visible in the HI map of NGC 4522 (Kenney et al. 2004). According to Kenney et al. (2004, Fig. 4) region (1) resides in extraplanar gas that was stripped out and removed from the disk via ram pressure. The extraplanar HII regions (2) and (3) lie in NGC 3628 and have projected distances from the midplane of their host galaxy of $2.8 \mathrm{kpc}$ and $3.0 \mathrm{kpc}$, respectively. We use the oxygen abundance $12+\log (\mathrm{O} / \mathrm{H})$ to derive metallicities. Oxygen is widely used as a reference element because it is relatively abundant, emits strong lines in the optical (as an important cooling element), and is observed in many ionization states (Kewley \& Dopita 2002). With the aid of excitation models, oxygen abundances can also be used to derive electron temperatures. There are different ways to derive oxygen abundances and temperatures. Direct methods use ratios of auroral and nebular lines as they are temperature sensitive; (e.g., the auroral line [OIII] $\lambda 4363$ in combination with [OIII] $\lambda \lambda 4959,5007)$. The line intensities of the auroral lines in spectra are weak, especially at solar metallicities, or higher. We did not detect any of these weak lines. Our spectra contain the strong emission lines such as the Balmer lines, [OII] $\lambda \lambda 3726,3729,[\mathrm{OIII}] \lambda \lambda 4959,5007$, and $[\mathrm{NII}] \lambda \lambda 6548,6583$. Owing to the spectral resolution, the lines of [O II] $\lambda 3726.0$ and [O II] $\lambda 3728.8$ were blended in the spectra and hence we use the sum of the doublet lines. To derive oxygen abundances, we use strong-line calibrations. There are different strong-line calibration types, i.e., empirical (e.g., Pilyugin 2001; Pilyugin \& Thuan 2005; Pettini \& Pagel 2004; Pilyugin et al. 2014) and theoretical (e.g., Kewley \& Dopita 2002; Kobulnicky \& Kewley 2004), which show well-known offsets between 0.1 and 0.7 dex (Stasińska 2002; Modjaz et al. 2008; Moustakas et al. 2010). The empirical methods use a fitted relationship between direct metallicities and strong-line ratios in the optical of a sample of HII regions to derive metallicity relations while the theoretical methods use photoionization models such as Cloudy (Ferland et al. 1998) to predict how theoretical emission line ratios depend on the input metallicity. We use three different calibrations, investigate their offsets, and discuss possible reasons. Using our measurements of the oxygen abundances, we explore the origin of the extraplanar HII regions.

The data reduction and observational strategy is given in Sect. 2. We then describe the measurement of the emission lines and the different calibration types for the analysis in Sect. 3. Section 4 contains the results. In Sect. 5 a short summary and the conclusions are given.

\section{Data}

\subsection{Spectroscopic data}

The spectroscopic data were obtained at the European Southern Observatory (ESO) La Silla with ESO Faint Object Spectrograph and Camera (EFOSC2 v.2) attached to the $3.6 \mathrm{~m}$ telescope during the nights of March 7-9, 2000. A $2048 \times 2048$ CCD detector with a pixel size of $15 \mu \mathrm{m}$ was used. The field of view is $5.4^{\prime} \times$ $5.4^{\prime}$ with a pixel scale of $0.316^{\prime \prime} /$ pixel. With the two different grisms 7 (3270-5240 $\AA$ ) and 9 (4700-6700 ̊), we were able to detect the important lines in the optical. The measured spectral resolution is $6.7 \AA$ in both grisms. The slit had a length of $5^{\prime}$ and a width of $1^{\prime \prime}$. The seeing was around $1.1^{\prime \prime}$ (see Table 1). The long slit was oriented differently in the galaxies. The slit positions for the galaxies NGC 3628 and NGC 4522 are shown in Figs. 1 and 2 and we report the position angles in Table 1. The slit position was chosen to go through both extraplanar regions and the disk. The total integration time for NGC 3628 was one hour in each grism. For NGC 4522, two slit positions were observed with the first covering the extraplanar region (s1) and the second the galaxy (s2). The total integration time was $20 \mathrm{~min}$ in 

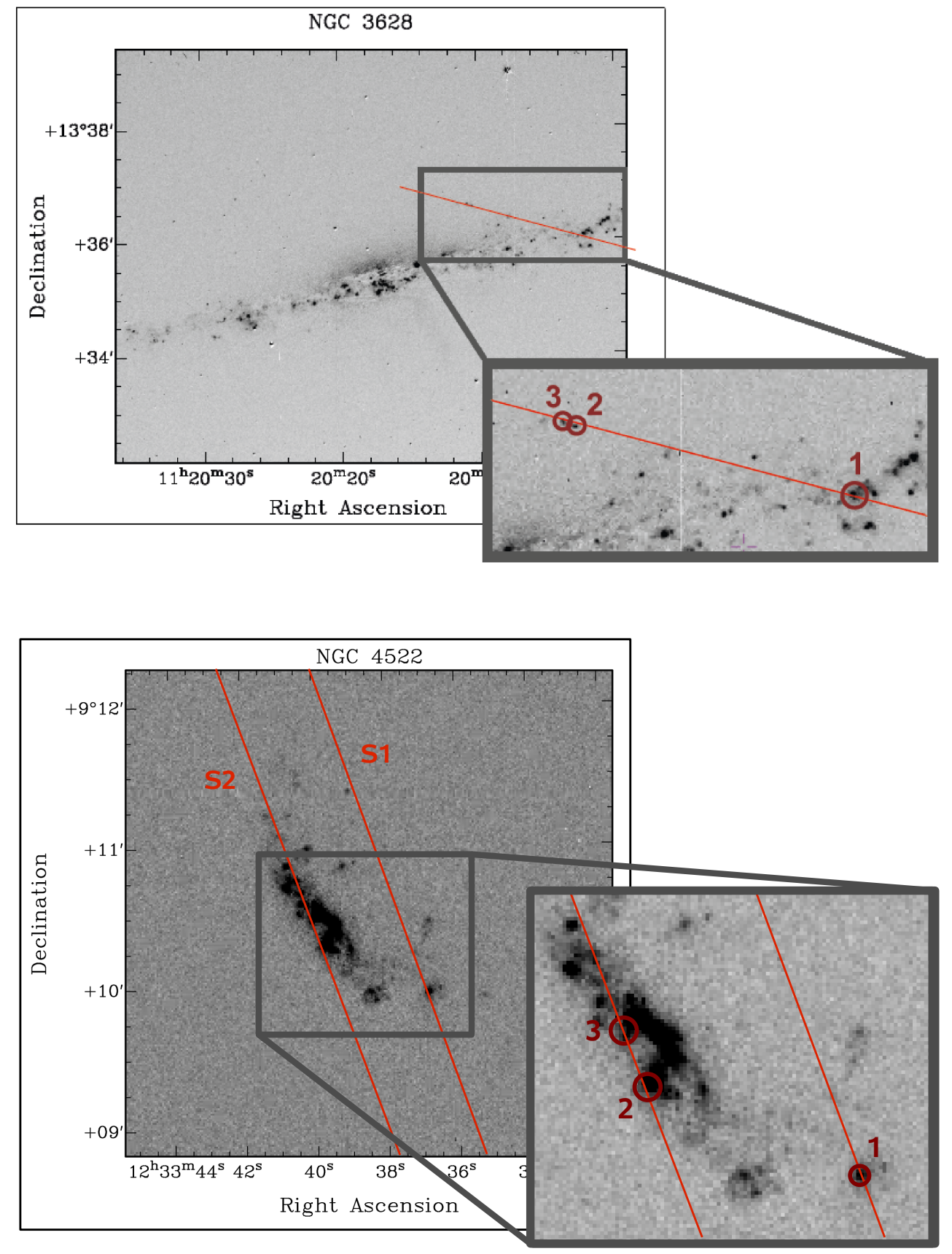

Fig. 1. Slit position on an $\mathrm{H} \alpha$ image of NGC 3628. The marked regions are analyzed.
Fig. 2. Slit position on an $\mathrm{H} \alpha$ image of NGC 4522. The marked regions are analyzed. slit position s1 with grism 7 and slit position s2 with both grisms. Slit position s1 with grism 9 was observed with two exposures of 20 min each (see Table 1 for details of the observations).

The data reduction was carried out using the image reduction and analysis facility (IRAF, Tody 1986, 1993). This includes the corrections for bias and overscan, flat-field response, and cosmic rays with L.A. Cosmic (van Dokkum 2001). Additionally, the wavelength calibration was achieved with helium-argon spectra and the flux calibration was carried out with standard stars (EG274, LTT2415). In order to obtain the pure emission from the HII regions, night sky background emission above and below these regions was averaged and subtracted with the IRAF task background. There are some residuals at $6300 \AA$ in the spectrum of NGC 4522 grism 9 left, which do not influence the data analysis. The galaxy continuum was subtracted by the IRAF task continuum, which was used with a linear spline 12th order and finally the spectra were coadded. Figures 3, 4 show the resulting spectra. To correct for stellar absorption, the non-continuumsubtracted spectra were coadded. All listed flux spectral densities are taken from these non-continuum-subtracted spectra.

\subsection{Narrowband imaging}

The $\mathrm{H} \alpha$ imaging for the galaxies discussed in this paper was generated from two different sources: NGC 3628 was observed on May 8, 1991 using the ESO New Technology Telescope (NTT, red arm of the ESO Multi-Mode Instrument (EMMI)) for $900 \mathrm{~s}$ in $\mathrm{H} \alpha$ and $300 \mathrm{~s}$ in $\mathrm{R}$ as part of ESO program 047.01-003. The detector was a Ford $2048^{2}$ single CCD (ESO CCD \#24). Both images were taken with subarcsecond seeing. The data and all relevant calibration files taken during the run and a few days before and after the observing run were retrieved from the ESO archive and re-reduced by us using IRAF in the usual manner. L.A. Cosmic (van Dokkum 2001) was used to clear the image 
NGC 3628 Grism7

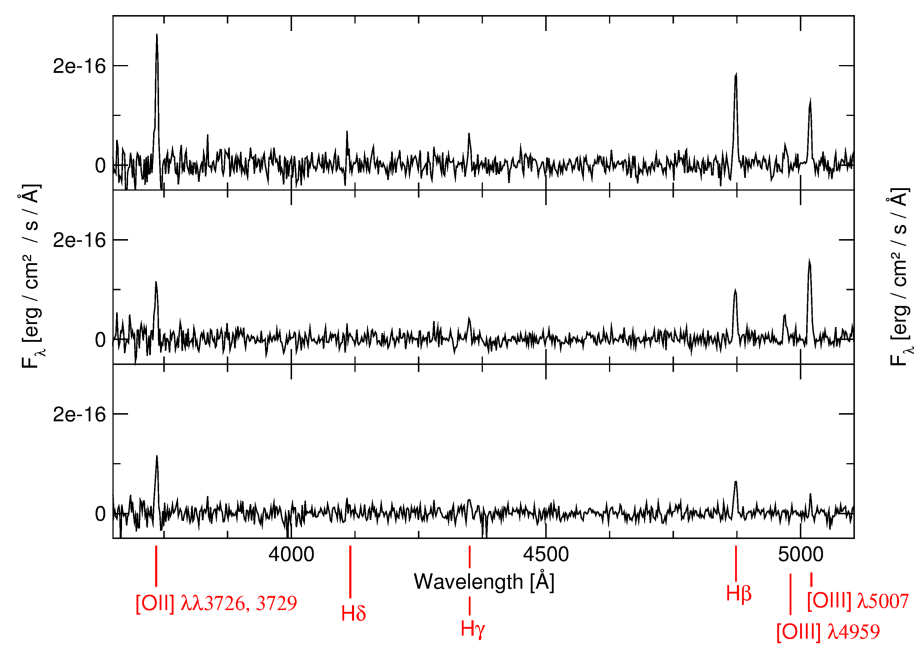

Fig. 3. Spectrum of NGC 3628 (grism 7 and grism 9).
NGC 3628 Grism9

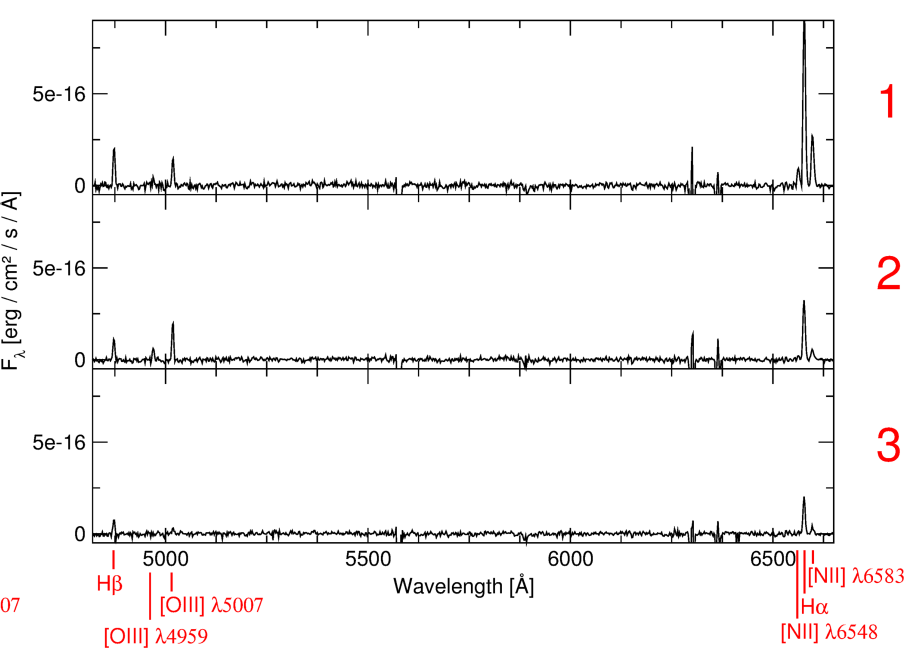

NGC 4522 Grism7

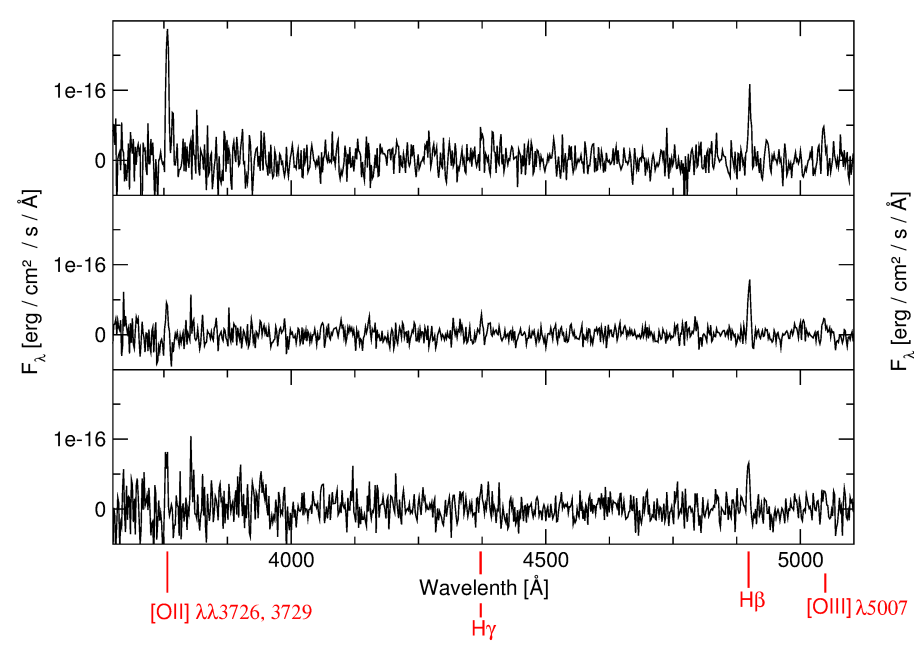

Fig. 4. Spectrum of NGC 4522 (grism 7 and grism 9).

of cosmic rays to produce the final $\mathrm{H} \alpha$ and final $R$ image. The continuum subtracted image was then produced by aligning $\mathrm{H} \alpha$ and $R$ band, homogenizing the PSF, and subtracting the appropriately scaled $\mathrm{R}$ image from the $\mathrm{H} \alpha$ image (e.g., Skillman et al. 1997). To determine the scaling factor, we measured the apparent fluxes for several stars in the field on both the $\mathrm{H} \alpha$ and $R$ images. Scaling, subtraction, and correction to reach an optimal correction of the continuum light of the galaxy was performed with our own IRAF scripts. The NGC $4522 \mathrm{H} \alpha$ data were taken directly from the work of Koopmann et al. (2001) and the Galaxy On Line Database Milano Network (GOLDMine, Gavazzi et al. 2003). The images were astrometrically calibrated to the Digitized Sky Survey (DSS) system. Flux calibration of the continuum subtracted $\mathrm{H} \alpha$ images was performed by transferring the total Sloan Digital Sky Survey (SDSS) $r^{\prime}$ band flux of each galaxy to the its continuum image. With the derived scaling factor (see above), the flux scale can then be directly transferred to the continuum corrected $\mathrm{H} \alpha$ image. A conversion from magnitudes to flux was performed using the fact that SDSS is calibrated in $\mathrm{AB}$ magnitudes, so that the zero-point flux density of each filter is $3631 \mathrm{Jy}$ (with $1 \mathrm{Jy}=1$ Jansky $=$ $\left.10^{-26} \mathrm{~W} \mathrm{~Hz}^{-1} \mathrm{~m}^{-2}=10^{-23} \mathrm{erg} \mathrm{s}^{-1} \mathrm{~Hz}^{-1} \mathrm{~cm}^{-2}\right)^{1}$.

\section{Analysis}

\subsection{Emission line measurements}

The spectra were analyzed using the splot task in IRAF. We chose the size of the extraction region of the extraplanar and disk HII regions depending on the extent of their $\mathrm{H} \alpha$ emission along the spatial axis in the spectra. The emission line flux densities were measured with the deblending tool of IRAF/splot, with which we fit Gaussians to obtain fluxes and central peak positions. We used the overlapping wavelength range of the two grisms for putting the spectra on a common flux scale. Unfortunately, the straight calibration showed different absolute fluxes of the emission lines due to differences in sky transparency conditions. Thus, it was necessary to scale the fluxes of the two grisms

1 http://classic.sdss.org/dr7/algorithms/fluxcal.html 


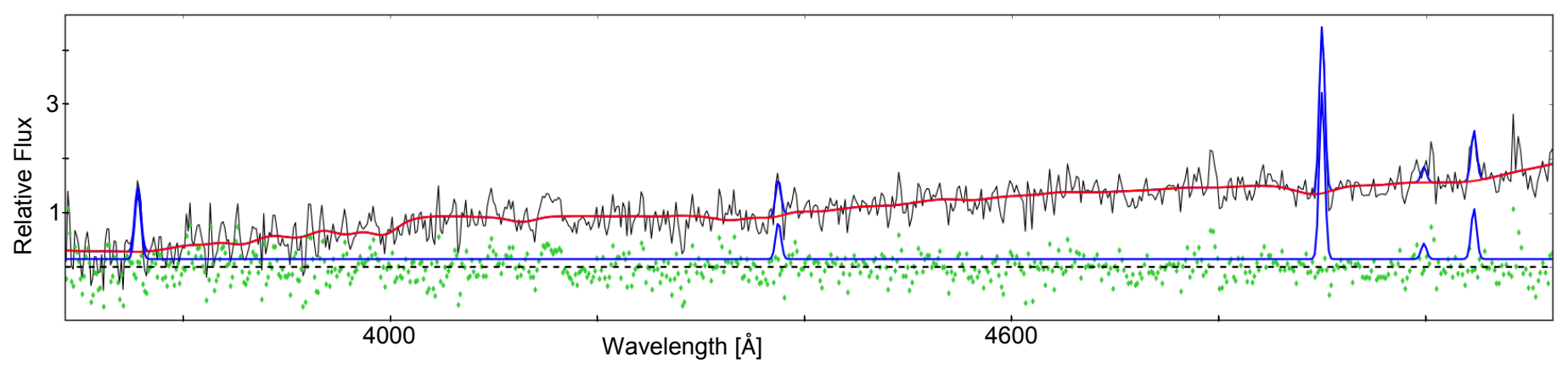

Fig. 5. pPXF spectrum of region (2) of NGC 4522 (grism 7). The black line represents the observed spectrum; the blue line shows the emission lines fitted by pPXF to the observed spectrum along with the extracted pure emission line spectrum. The red line is the fitted continuum to the data and the green dots are the residuals around the zero value (gray dashed line).

Table 2. HII region properties.

\begin{tabular}{clcclcc}
\hline \hline Galaxy & Region & $\mathrm{H} \beta$ grism 7 & $\mathrm{H} \beta$ grism 9 & $c$ & $v_{\text {helio }}\left[ \pm 23 \mathrm{~km} \mathrm{~s}^{-1}\right]$ & Size HII region [pc] \\
\hline NGC 3628 & 1 & $(4.63 \pm 0.67) \times 10^{-16}$ & $(4.31 \pm 0.40) \times 10^{-16}$ & 0.95 & 671 & \\
& $2(\mathrm{e})$ & $(3.89 \pm 0.56) \times 10^{-16}$ & $(3.89 \pm 0.36) \times 10^{-16}$ & $0.12^{(1)}$ & 648 & $150 \pm 30$ \\
& $3(\mathrm{e})$ & $(2.53 \pm 0.36) \times 10^{-16}$ & $(2.53 \pm 0.23) \times 10^{-16}$ & $0.00^{(2)}$ & 650 & $150 \pm 30$ \\
NGC 4522 & $1(\mathrm{e})$ & $(6.22 \pm 0.90) \times 10^{-16}$ & $(6.12 \pm 0.56) \times 10^{-16}$ & 0.30 & 2366 & $325 \pm 40$ \\
& 2 & $(1.22 \pm 0.18) \times 10^{-15}$ & $(1.35 \pm 0.12) \times 10^{-15}$ & 0.33 & 2320 & \\
& 3 & $(5.67 \pm 0.82) \times 10^{-16}$ & $(6.04 \pm 0.56) \times 10^{-16}$ & 0.37 & 2253 & \\
\hline
\end{tabular}

Notes. $\mathrm{H} \beta$ fluxes in erg $\mathrm{cm}^{-2} \mathrm{~s}^{-1}$; (e) = extraplanar. ${ }^{(1)}$ No EW correction applied; ${ }^{(2)}$ No EW correction, and no reddening correction applied.

to one another first. The common lines of $\mathrm{H} \beta$ and [OIII] $\lambda 5007$ were used for this in grism 7 and grism 9.

\subsection{Stellar continuum and reddening correction}

Since absorption lines in the underlying stellar continuum can affect the measurement of the emission lines (e.g., the Balmer lines), we need to investigate this effect in our spectra. We identified a weak contribution of underlying stellar continuum in the disk HII regions, which was even smaller in the extraplanar HII region (1) of NGC 4522 in our spectra. We corrected the $\mathrm{H} \alpha$ and $\mathrm{H} \beta$ lines for absorption with $2 \AA$ in the equivalent width (EW; Moustakas et al. 2010). There were no metal line absorption features visible in the stellar continuum of the disk HII regions and therefore it was not possible to perform a reliable population synthesis, for example, with STARLIGHT (Cid Fernandes et al. 2004). Nevertheless, we tried pPXF fitting (Cappellari \& Emsellem 2004; Cappellari 2017) for one HII region. This routine does full spectral fitting to extract stellar and gas kinematics and stellar populations information simultaneously (Cappellari 2017). This was performed for the disk HII region (2) in grism 7 of NGC 4522 to be sure that our applied standard absorption correction is reliable. As Fig. 5 shows, the pPXF-predicted absorption features are very weak. The resulting corrected line ratio of $\mathrm{H} \gamma / \mathrm{H} \beta$ from $\mathrm{pPXF}$ was 0.26 and, after dereddening, 0.29, which is at odds with the theoretical value of 0.46 . Clearly, because of the missing information of the metal-absorption features, the pPXF code cannot find a good solution for our data. Therefore, a first order EW correction of the Balmer lines seems to be the best option. There was no continuum visible in the extraplanar HII regions (2), (3) of NGC 3628 and therefore no EW correction was applied, which is similar to the approach taken for the analysis of faint high redshift galaxies (e.g., Mannucci et al. 2009). To account for extinction, the fluxes were dereddened with the IRAF task redcorr. For this we used the theoretical Balmer decrement of $\mathrm{H} \alpha / \mathrm{H} \beta=2.87$ (Case B, $10000 \mathrm{~K}$ ), the Galactic extinction law $f(\lambda)$ from Savage \& Mathis (1979) and the extinction parameter c (see Table 2) with the following equation used in IRAF:

$I_{\text {corr }}(\lambda)=I_{\mathrm{obs}}(\lambda) \times 10^{c} \cdot f(\lambda)$,

where $I_{\text {corr }}(\lambda)$ is the corrected flux and $I_{\text {obs }}(\lambda)$ the observed flux. To judge the effect of the chosen extinction law, we tested our reddening correction using the LMC extinction curve (Howarth 1983). For the $\mathrm{H} \alpha / \mathrm{H} \beta$ line ratio, the change is $0.3 \%$, whereas the biggest change is $3.5 \%$ with the $[\mathrm{OII}] / \mathrm{H} \beta$ line ratio. We claim the reddening correction is only weakly dependent on the adopted reddening curve. If possible, the lines were measured in grism 9.

The $\mathrm{H} \beta$ fluxes are listed in Table 2. The dereddened and scaled fluxes relative to $\mathrm{H} \beta$ are listed in Table 3 .

\subsection{Errors}

\subsubsection{Flux errors}

Errors in the emission line fluxes can arise from the calibration and from the measurement of the emission lines with the task splot in IRAF. Statistical errors are calculated within splot. These errors are explained and discussed below.

- The error in the flux calibration was tested by applying the calibration to a standard star and comparing the resultant fluxes with the known fluxes. The error was found to be $12 \%$ in grism 7 and $6 \%$ in grism 9. 
Table 3. Dereddened and scaled fluxes normalized to $\mathrm{H} \beta$.

\begin{tabular}{clcccccccc}
\hline \hline Galaxy & Region & {$[\mathrm{OII}] \lambda \lambda 3726,3728$} & $\mathrm{H} \gamma$ & {$[\mathrm{OIII}] \lambda 4959$} & {$[\mathrm{OIII}] \lambda 5007$} & {$[\mathrm{NII}] \lambda 6548$} & $\mathrm{H} \alpha$ & {$[\mathrm{NII}] \lambda 6583$} \\
\hline NGC 3628 & 1 & $1.85 \pm 0.20$ & $0.48 \pm 0.09$ & $0.14 \pm 0.04$ & $0.31 \pm 0.05$ & $0.27 \pm 0.04$ & $2.87 \pm 0.31$ & $0.82 \pm 0.12$ \\
& 2 (e) & $1.11 \pm 0.16$ & $0.46 \pm 0.09$ & $0.52 \pm 0.11$ & $1.57 \pm 0.25$ & $0.16 \pm 0.03$ & $2.87 \pm 0.31$ & $0.50 \pm 0.07$ \\
& 3 (e) & $1.71 \pm 0.32$ & $0.40 \pm 0.11$ & - & $0.30 \pm 0.05$ & $0.17 \pm 0.03$ & $2.84 \pm 0.31^{(1)}$ & $0.54 \pm 0.08$ \\
NGC 4522 & 1 (e) & $2.02 \pm 0.32$ & $0.45 \pm 0.11$ & - & $0.36 \pm 0.08$ & $0.17 \pm 0.04$ & $2.87 \pm 0.31$ & $0.70 \pm 0.10$ \\
& 2 & $0.88 \pm 0.14$ & $0.40 \pm 0.10$ & $0.24 \pm 0.03$ & $0.38 \pm 0.07$ & $0.28 \pm 0.04$ & $2.87 \pm 0.31$ & $0.95 \pm 0.13$ \\
& 3 & $0.89 \pm 0.16$ & - & $0.09 \pm 0.01$ & $0.31 \pm 0.05$ & $0.35 \pm 0.06$ & $2.87 \pm 0.31$ & $0.85 \pm 0.13$ \\
\hline
\end{tabular}

Notes. $(\mathrm{e})=$ extraplanar, ${ }^{(1)}$ no dereddening applied.

- The task splot itself displayed errors after specifying the parameters inverse gain and noise $\sigma_{0}$. For each emission line, the error was determined separately. We tested these errors with the [OII] $\lambda \lambda 3726,3728$ and [OIII] $\lambda 5007$ lines in grism 7 and with $\mathrm{H} \alpha$ and [OIII] $\lambda 5007$ lines in grism 9. By repeated measurements of the same emission line with variations in applying the deblending tool within the splot task, for example, for variations in the initial estimate of the peak position and continuum, the resulting errors were found to be similar to the splot errors. Therefore the splot errors were used for further error propagation.

The resulting flux error (sum of the squares) for $\mathrm{H} \beta$ is $14.4 \%$ in grism 7 and $9.2 \%$ in grism 9 . The errors are propagated throughout the complete abundance analysis (Gaussian error propagation). To calculate the final error in the oxygen abundance determination, we added the errors of the different calibrations to the flux error (sum of squares).

\subsubsection{Wavelength errors}

The errors of the velocity determination result from the wavelength calibration and the measurement of the peak positions of the emission lines, which are given below in more detail.

- The applied wavelength calibration function had a dispersion of $0.5 \AA$, which was taken as the error of the calibration. This was further checked by measuring the position of three sky lines per grism and comparing these with values in the literature (Slanger et al. 2003). The error was confirmed to be $0.5 \AA$.

- The error of the peak positions was determined by measuring four times the same emission line with variation in the initial estimate of the peak position using the deblending tool within the splot task. The average of the errors given by splot was taken as the resulting error.

The total error in wavelength is $2.13 \AA$ in grism 7 , which mainly originates from the high splot errors due to the low signalto-noise ratio $(\mathrm{S} / \mathrm{N})$ in this grism. The total wavelength error is $0.50 \AA$ in grism 9 .

\subsection{Parameter determination}

The projected distances from the extraplanar regions to the host galaxy were determined as the projected height perpendicular to the central line of the disk, which was defined by an isophotal fit to the $3.6 \mu \mathrm{m}$ image for NGC 4522 and by the central emission in the $\mathrm{H} \alpha$ image for NGC 3628. The heliocentric velocities of the HII regions were determined from the redshifts of the $\mathrm{H} \alpha$ line, which is the brightest line. The heliocentric correction was calculated by the IRAF task rvcorrect.

The metallicities were derived using the oxygen abundance $12+\log (\mathrm{O} / \mathrm{H})$ by the following three different methods.

\subsubsection{Method 1}

Kewley \& Dopita (2002; theoretical) use stellar population synthesis and photoionization models to produce a set of ionization parameter and abundance diagnostics based on the use of strong optical emission lines. These authors claim the ratio of [NII] $\lambda 6583$ to [OII] $\lambda \lambda 3726,3729$ is a reliable diagnostic because it is independent of the ionization parameter and has no local maximum like the $R_{23}$ parameter. Additionally, the [NII], [OII] lines are relatively strong, even in low $\mathrm{S} / \mathrm{N}$ spectra. With $R=I([\mathrm{NII}] \lambda 6583) / I([\mathrm{OII}] \lambda \lambda 3726,3729)$ a first estimate of the oxygen abundance is calculated

$$
\begin{aligned}
& 12+\log (\mathrm{O} / \mathrm{H})= \\
& \quad \log \left(1.54020+1.26602 R+0.167977 R^{2}\right)+8.93
\end{aligned}
$$

Following that paper, the oxygen abundance is taken as final if the result is $12+\log (\mathrm{O} / \mathrm{H}) \geq 8.6$, which was the case in all regions discussed in this paper.

Limitations: Kewley \& Dopita (2002) mention that the observed $[\mathrm{NII}] /[\mathrm{OII}]$ abundance ratio shows large scatter from object to object due to the varying age distribution of the stellar population for a sample of HII regions. Furthermore, in this method, the oxygen abundance depends only on the $[\mathrm{NII}] /[\mathrm{OII}]$ ratio, which is taken from an empirical fit to the observed behavior of the $[\mathrm{NII}] /[\mathrm{OII}]$ ratio in HII regions. Because the lines are well separated in wavelength, this method uses one line from each grism and hence it is highly dependent on the scaling and reddening correction.

We also used the ionization parameter, which was determined empirically (Kewley \& Dopita 2002), as follows:

$$
\begin{aligned}
& \log (I([\mathrm{OIII}]) /(I([\mathrm{OII}]))= \\
& k_{0}+k_{1} \log (q)+k_{2} \log (q)^{2}+k_{3} \log (q)^{3},
\end{aligned}
$$

with given coefficients for $12+\log (\mathrm{O} / \mathrm{H})=8.9 k_{0}=-52.6367$, $k_{1}=16.088, k_{2}=-1.67443$ and $k_{3}=0.0608004$.

\subsubsection{Method 2}

Pilyugin et al. (2014) is a revised version of Pilyugin (2001), Pilyugin \& Thuan (2005). They calibrated empirically the $R_{3}$ 
Table 4. $[\mathrm{OIII}] / \mathrm{H} \beta$ and ionization parameter q of NGC 3628.

\begin{tabular}{clcc}
\hline \hline Galaxy & Region & {$[\mathrm{OIII}] / \mathrm{H} \beta$} & $q$ (Kewley \& Dopita 2002) \\
\hline NGC 3628 & 1 & $0.31 \pm 0.05$ & $(1.95 \pm 0.23) \times 10^{7}$ \\
& $2(\mathrm{e})$ & $1.57 \pm 0.25$ & $(2.68 \pm 0.30) \times 10^{8}$ \\
& $3(\mathrm{e})$ & $0.30 \pm 0.05$ & $(1.67 \pm 0.16) \times 10^{7}$ \\
\hline
\end{tabular}

Notes. $(\mathrm{e})=$ extraplanar.

and $P$ (excitation parameter) against the oxygen abundances determined with temperature-sensitive lines (direct $T_{\mathrm{e}}$ method) of more than $250 \mathrm{HII}$ regions from 3904 different spectra of 130 late-type galaxies, providing central oxygen abundances and abundance gradients. This is carried out for low metallicities $(12+\log (\mathrm{O} / \mathrm{H}) \leq 8.0)$ (Pilyugin 2000) and high metallicities $(12+\log (\mathrm{O} / \mathrm{H}) \geq 8.3)$. In this paper the relation for the higher metallicities (upper branch: $12+\log (\mathrm{O} / \mathrm{H}) \geq 8.3$ ) is used.

The excitation parameter is defined as $P=R_{3} / R_{23}$ whereas

$R_{3}=I([\mathrm{OIII}] \lambda \lambda 4959,5007) / I(\mathrm{H} \beta)$

$R_{23}=\frac{I([\mathrm{OII}] \lambda \lambda 3726,3729)+I([\mathrm{OIII}] \lambda \lambda 4959,5007)}{I(\mathrm{H} \beta)}$.

Using this definition of the excitation parameter $P$ and $R_{3}$, they computed

$$
\begin{aligned}
12+\log (\mathrm{O} / \mathrm{H})= & 8.334+0.533 P \\
& -(0.338+0.415 P) \log R_{3} \\
& -(0.086-0.225 P)\left(\log R_{3}\right)^{2} .
\end{aligned}
$$

The oxygen abundance was determined with Eq. (6).

Limitations: in the intermediate-metallicity regime, $12+\log (\mathrm{O} / \mathrm{H}) \sim 8.0-8.4$, the derived oxygen abundance is highly affected by the $R_{23}$ ambiguity, therefore the average value of the higher and lower metallicity regime should be assumed (López-Sánchez et al. 2012).

\subsubsection{Method 3}

Pettini \& Pagel (2004) empirically calibrated the parameter O3N2 (Eq. (7)) against the oxygen abundances of a sample of 137 extragalactic HII regions determined mostly from temperature-sensitive lines (direct $T_{\mathrm{e}}$ method) or photoionization models. This results in the oxygen abundance determination via a linear fit between -1 and 1.9 , which is used in this paper (Eq. (8)), as follows:

$$
\begin{gathered}
\mathrm{O} 3 \mathrm{~N} 2=\log \left(\frac{I([\mathrm{OIII}] \lambda 5007) / I(\mathrm{H} \beta)}{I([\mathrm{NII}] \lambda 6583) / I(\mathrm{H} \alpha)}\right) \\
12+\log (\mathrm{O} / \mathrm{H})=8.73-0.32 \cdot \mathrm{O} 3 \mathrm{~N} 2
\end{gathered}
$$

Limitations: this method is only valid for $12+\log (\mathrm{O} / \mathrm{H}) \gtrsim 8.7$ (López-Sánchez et al. 2012).

\section{Results and discussion}

Tables 4 and 5 show the results of the analysis. In this section, we discuss the Balmer decrement, compare the calibrations, and describe the results of the individual galaxies and their HII regions.

\subsection{Balmer decrement of the HII regions}

The measured Balmer decrement of $\mathrm{H} \alpha / \mathrm{H} \beta$ from the extraplanar HII region (3) of NGC 3628 is slightly lower than the theoretical value. Possibly, the region has a higher temperature. Nevertheless, considering the error, the Balmer decrement fits the theoretical value for all regions. The extinction parameter of the extraplanar HII region (3) of NGC 3628 is zero. Therefore, no reddening correction was applied. As the two extraplanar regions (2) and (3) of NGC 3628 are away from the galaxy disk, they are expected to be only slightly influenced by reddening, which is consistent with our observation.

We did a sanity check on region (3) of NGC 3628 to test how sensitive the oxygen abundance is to the uncertainty in [OII] $\lambda \lambda 3726,3728$. The [OII] line in grism 7 is very important for method 1, however grism 7 does not provide high $\mathrm{S} / \mathrm{N}$. We determined the influence of this line on the abundance measurement. We calculated the oxygen abundance with method 1 using the measured value of $\mathrm{H} \alpha / \mathrm{H} \beta=2.84$ and additionally with considering the error of 0.31 , which leads to the upper limit of the $\mathrm{H} \alpha / \mathrm{H} \beta$ ratio of 3.15. The latter ratio would lead to an extinction parameter of $c=0.13$. That increases the [OII] $\lambda \lambda 3726,3728$ line ratio from 1.71 to 1.86 and decreases [NII] $\lambda 6583$ from 0.54 to 0.49 . The resulting oxygen abundance of method 1 would change from 8.92 to 8.87 . Thus, the oxygen abundance determination is robust toward our errors and the analysis is therefore reliable.

\subsection{Oxygen abundances: general trends and comparison of the calibrations}

The results of the abundance determination are consistent within all regions.

In general, method 1 (Kewley \& Dopita 2002) shows the highest values of $12+\log (\mathrm{O} / \mathrm{H})$ while method 2 (Pilyugin et al. 2014) and method 3 (Pettini \& Pagel 2004) provide lower values. The mean discrepancy between method 1 and method 2 is 0.41 dex. The mean offset between method 1 and method 3 0.36 dex and between method 2 and method 3 is 0.05 dex. This result is in agreement with other analyses (Modjaz et al. 2008; Kewley \& Ellison 2008).

To check the determined oxygen abundances, we searched for reference metallicities of the galaxies. For NGC 3628, an oxygen abundance of $12+\log (\mathrm{O} / \mathrm{H})=8.57$ was determined by Engelbracht et al. (2008) using an integrated spectrum of the galaxy and empirical strong-line methods (Pettini \& Pagel 2004; Pilyugin \& Thuan 2005). To compare to their value, we also calculated the mean of the methods. For the disk HII region (1) of NGC 3628, the reference oxygen abundance is similar to the mean values of the two calibrations of 8.56 dex.

The comparison of theoretical and empirical strong-line calibrations show well-known offsets (Stasińska 2002; Modjaz et al. 2008; Moustakas et al. 2010). We confirm the offsets with a mean difference of 0.37 dex.

Strong-line calibrations use the fact that the metallicity in large HII regions is connected to the mean effective temperature of the stellar radiation field and the ionization parameter (Stasińska 2010). The theoretical calibrations use photoionization models to derive metallicities. These models are limited and depend strongly on the temperature structure calculations of the nebula (Kewley \& Ellison 2008). This leads to discrepancies of up to 0.2 dex within the theoretical calibrations and up to 0.6 dex in comparison to empirical methods. Empirical calibrations use the relationship between direct metallicities and 
Table 5. Resulting oxygen abundances of the different methods.

\begin{tabular}{clccc}
\hline \hline \multirow{2}{*}{ Galaxy } & \multirow{3}{c}{ Region } & Method 1 & Method 2 & Method 3 \\
\cline { 3 - 5 } & & Kewley \& Dopita $(2002)$ & Pilyugin et al. (2014) & Pettini \& Pagel (2004) \\
& & {$[ \pm 0.04]$} & {$[ \pm 0.10]$} & {$[ \pm 0.14]$} \\
\hline \multirow{2}{*}{ NGC 3628 } & 1 & $8.96 \pm 0.06$ & $8.58 \pm 0.11$ & $8.72 \pm 0.15$ \\
& $2(\mathrm{e})$ & $8.94 \pm 0.07$ & $8.43 \pm 0.14$ & $8.42 \pm 0.21$ \\
& $3(\mathrm{e})$ & $8.92 \pm 0.07$ & $8.60 \pm 0.11$ & $8.67 \pm 0.15$ \\
\hline \multirow{2}{*}{ NGC 4522 } & 1 (e) & $8.92 \pm 0.07$ & $8.54 \pm 0.13$ & $8.63 \pm 0.16$ \\
& 2 & $9.13 \pm 0.04$ & $8.68 \pm 0.12$ & $8.69 \pm 0.15$ \\
& 3 & $9.12 \pm 0.04$ & $8.69 \pm 0.11$ & $8.72 \pm 0.15$ \\
\hline
\end{tabular}

Notes. (e) = extraplanar, the error in the heading is the intrinsic error of the calibration according to the publication.

strong-line ratios of a sample of HII regions. This sample shows special characteristics in densities, the strength of the radiation field, and the state of ionization (Stasińska 2010). Therefore, empirical methods should be used with HII regions that are comparable to the calibration sample. One origin of discrepancies within various empirical methods is the variety of characteristics of HII regions. A possible reason for the offset in the comparison to theoretical calibrations is temperature fluctuations in HII regions. In metal-rich HII regions effective cooling processes could lead to strong temperature gradients (Garnett 1992). Therefore the temperature determined with the forbidden lines is overestimated and the oxygen abundance is underestimated (Garnett 1992). With knowledge of the fluctuations, the oxygen abundance would shift 0.2-0.3 dex (Moustakas et al. 2010). Another possible reason has been mentioned by Nicholls et al. (2012). If the electrons in HII regions do not follow a Maxwell-Boltzmann equilibrium energy distribution but follow a non-equilibrium kappa distribution, it should be possible to estimate the temperature and metallicity in these regions more accurately.

In addition to the calibrations we used in this paper, Kewley \& Ellison (2008) also investigated seven other calibrations and got a mean difference of up to 0.7 dex between theoretical and empirical calibrations. These authors propose to use oxygen abundance calibration with caution and to always specify the adopted calibration. They refer to relative oxygen abundances as a reliable tool, especially with the calibrations of Kewley \& Dopita (2002) and Pettini \& Pagel (2004).

\subsection{Individual galaxies}

In this section the individual galaxies with their extraplanar HII regions are presented. In interpreting our results, we account for the metallicity gradients in spiral galaxies (e.g., Garnett 1998; Sánchez-Menguiano et al. 2016), which can often change the metallicity by up to 0.3 dex within $R_{25}$ and does not depend on the different metallicity calculations (e.g., Magrini et al. 2011).

\subsubsection{NGC 3628}

NGC 3628 is a member of the Leo triplet and interacts with NGC 3627. The two analyzed extraplanar HII regions are located in a filament of NGC 3628 visible in HI (Wilding et al. 1993), which was probably formed due to tidal interactions. The regions have projected distances of $2.8 \mathrm{kpc}$ (2) and $3 \mathrm{kpc}$ (3).
Radial velocity: the determined radial velocities of the extraplanar HII regions are $648 \mathrm{~km} \mathrm{~s}^{-1}$ of region (2) and $650 \mathrm{~km} \mathrm{~s}^{-1}$ of region (3). Comparing this result to an HI velocity map of Wilding et al. (1993), the velocities are consistent. The same is true for the disk HII region (1). It is also obvious that the analyzed extraplanar regions (2), (3), and the disk region (1) are located in the same section of the velocity field. The extraplanar regions of NGC 3628 are located in a filament, which was probably formed by an interaction with NGC 3627. The velocity gradient within the filament as seen in the HI map is a hint that it has its origin in the outer parts of the galaxy. Therefore, the extraplanar regions and the disk region have comparable velocities within the rotation curve. The measured heliocentric velocities from our spectra confirm this (Table 2). There is a difference of only $20 \mathrm{~km} \mathrm{~s}^{-1}$ between the disk region and the extraplanar regions. Therefore, the extraplanar regions are comparable with the disk region in terms of metallicity gradients.

Metallicities: the derived oxygen abundances of the HII disk region (1) and extraplanar region (3) are the same within the errors in all methods. In method 1 the extraplanar region (3) has a slightly lower value than the disk region (1). Nevertheless, in general, region (3) seems to have a comparable abundance to the disk region (1). We conclude that this extraplanar region was formed in a tidal arm of disk material.

Region (2) of NGC 3628 shows the lowest value in method 3. This value is confirmed by method 2 . There is an indication that this region is highly ionized based on the high $[\mathrm{OIII}] / \mathrm{H} \beta$ ratio and the high ionization parameter in comparison to the other HII regions of this galaxy (see Table 4). In the diagnostic diagram (Fig. 6) the region shows the highest value of $\log (\mathrm{OIII} / \mathrm{H} \beta)$ of the HII regions analyzed in this paper but is still within the region of HII regions located.

\subsubsection{NGC 4522}

NGC 4522 is a member of the Virgo cluster. The optical distribution is undisturbed (Kenney \& Koopmann 1999) while the HI distribution is asymmetric (Kenney et al. 2004). There is indication that ram pressure of the Virgo cluster stripped the gas of the galaxy (Kenney et al. 2004). The extraplanar HII region (1) is located in the outer gas and has a projected distance of $1.4 \mathrm{kpc}$.

Radial velocity: the determined heliocentric velocities of the three HII regions are around $2310 \mathrm{~km} \mathrm{~s}^{-1}$. The extraplanar region (1) differs by $46 \mathrm{~km} \mathrm{~s}^{-1}$ from disk region (2) and by 


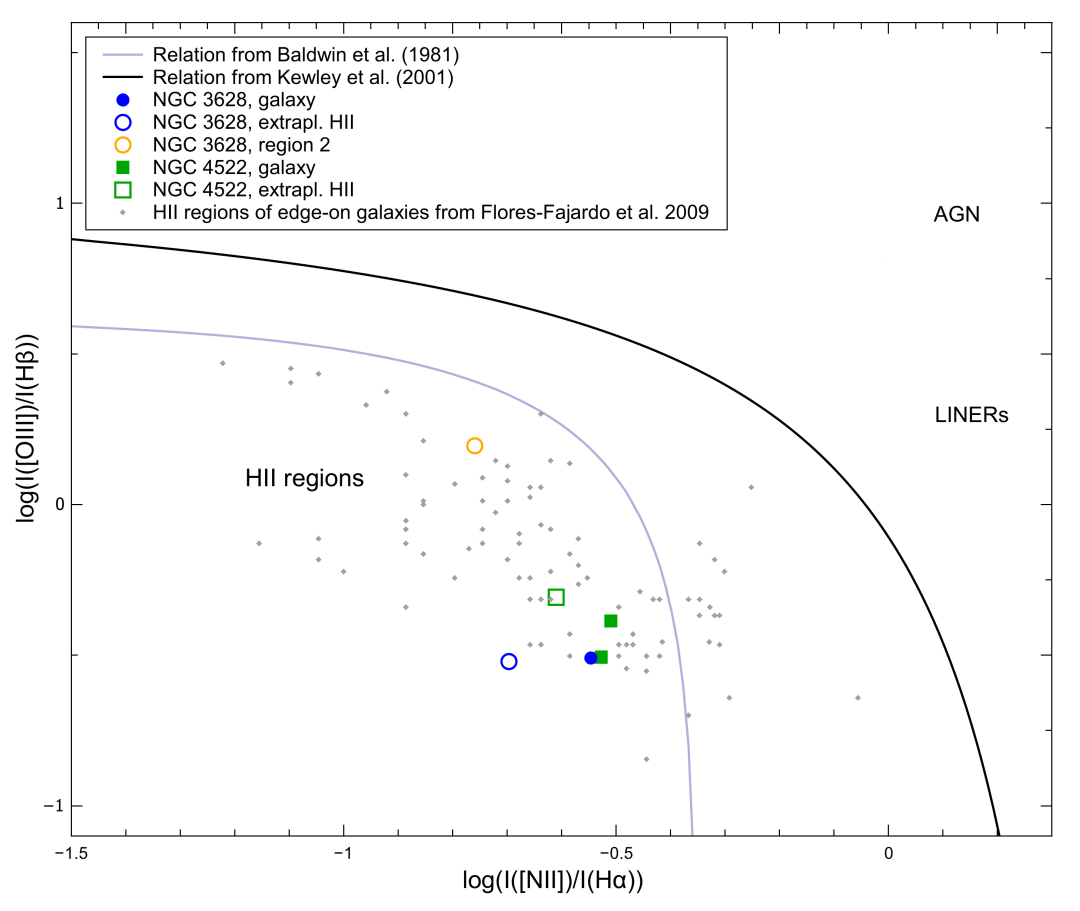

Fig. 6. Diagnostic diagram: lines are a fit through HII regions from Baldwin et al. (1981, gray) and the theoretical upper limit for the starburst model from Kewley et al. (2001, black). The errors of the six HII regions correspond to the size of the symbols. The gray diamonds are HII regions from a sample of edge-on galaxies from Flores-Fajardo et al. (2009).
$113 \mathrm{~km} \mathrm{~s}^{-1}$ from disk region (3) in velocity (Table 2). A similar trend is seen in the velocity map of Kenney et al. (2004) at the locations of the regions, but with values that are, on average, lower by $30 \mathrm{~km} \mathrm{~s}^{-1}$. One reason is that their central heliocentric velocity of $2337 \mathrm{~km} \mathrm{~s}^{-1}$ is offset from other measurements that are lower, for example, from HI of de Vaucouleurs et al. (1991b) at $2324 \pm 5 \mathrm{~km} \mathrm{~s}^{-1}$. As the $\mathrm{H} \alpha$ line lies at the edge of grism 9, we tested our measured heliocentric velocity using the [OIII] $\lambda 5007$ line, which lies in the middle of grism 9. We find on average $15 \mathrm{~km} \mathrm{~s}^{-1}$ higher heliocentric velocities, which is more in agreement with Kenney et al. (2004) and indeed is compatible within the errors.

Metallicities: the extraplanar HII region (1) shows lower oxygen abundances in comparison to the disk HII regions (2) and (3) in all methods; the mean difference is 0.17 dex. In method 3 the offset between disk and extraplanar HII regions is the smallest and not significant, whereas the offset of 0.20 dex in method 1 is the largest and most significant. Method 2 shows a slightly lower value. Even considering the combined errors, this is not significant either. However, considering all methods together the lower metallicity is slightly more significant than for method 1 alone. In the diagnostic diagram (Fig. 6), all regions are located close to each other. The Virgo Cluster galaxy NGC 4522 is close to peak ram pressure, where the outer gas disk has already been removed and the remaining gas disk is strongly truncated (Vollmer 2009). The slightly smaller oxygen abundance of the extraplanar HII region could be caused by the metallicity gradients that decrease the metallicity in the outer parts, which are the regions most affected by ram pressure stripping. We conclude that the extraplanar HII region (1) is located in these parts of stripped gas from the galaxy and therefore shows slightly lower metallicities.

\subsubsection{Origin of extraplanar H॥ regions}

The three analyzed extraplanar HII regions show different origins. These are tidal interactions in the galaxy NGC 3628 (tidal stream) and ram pressure in NGC 4522. These tital interactions are both influenced by the group or cluster environment.
As the extraplanar HII regions studied here show comparable or slightly lower oxygen abundances in comparison to the HII regions in the host galaxy, these extraplanar HII regions likely result from disk material. This result is different from the analysis of NGC 55 (Tüllmann et al. 2003; Kudritzki et al. 2016) in which two analyzed extraplanar HII regions show low oxygen abundance. Therefore, ejection from the disk was ruled out and Tüllmann et al. (2003) claimed that the regions were formed in the halo. The intergalactic regions of the Stephan's Quintet (Mendes de Oliveira et al. 2004) show a high oxygen abundance. These regions were probably formed in tidal tales of the four interacting galaxies. The extragalactic region of NGC 4402 (Cortese et al. 2004) seems also to have a high metallicity and thus is built with pre-enriched material. They ruled out a tidal stripping scenario due to the absence of a clear interaction signal. One possible scenario would be that the extragalactic HII region is formed by an interaction between NGC 4402 and the ICM of the Virgo. The mechanism of ram pressure stripping is probably present in the region of NGC 4388 (Oosterloo \& van Gorkom 2005). The same is true for the extraplanar HII region (1) of NGC 4522 analyzed in this paper, whereas the metallicity is low in the extraplanar region of NGC 4388 (Gerhard et al. 2002) and just slightly lower in our case.

\section{Summary and conclusions}

We present optical long-slit spectroscopic data of extraplanar HII regions and their edge-on host galaxies NGC 3628 and NGC 4522. In order to analyze these extraplanar HII regions, we determined metallicities (oxygen abundances). As the spectra contain strong emission lines such as the Balmer series, [OII] $\lambda \lambda 3726,3729$, [OIII] $\lambda \lambda 4959,5007$, and [NII] $\lambda \lambda 6548$, 6583 , we calculate the oxygen abundances of the extraplanar HII regions with different strong-line calibrations based on empirical data and theoretical photoionization grids. First, we probed the shift between the different types of strong-line calibrations (empirical and theoretical). We analyzed the extraplanar HII regions and at least one HII region located in the disk of the 
galaxies via three different calibrations (Kewley \& Dopita 2002; Pilyugin 2001; Pilyugin et al. 2014; Pettini \& Pagel 2004). Our analysis again confirms the known offsets with a mean value of 0.37 dex. Secondly, we investigated the origin of the extraplanar HII regions by comparing the oxygen abundance of the extraplanar and disk HII regions.

In NGC 3628 we derived comparable oxygen abundances, which leads to the conclusion that the extraplanar HII regions are formed from disk material. The two analyzed extraplanar HII regions (2) and (3) of NGC 3628 are located in a filament that emerges from the disk, which was probably formed from tidal interactions with NGC 3627.

The extraplanar HII region (1) of NGC 4522 shows slightly lower measured oxygen abundances in comparison to the disk HII regions (2) and (3). Therefore, ram pressure of the Virgo Cluster probably stripped out the gas of the galaxy. This outer gas, where the extraplanar HII region is located, is not as metal rich as the inner disk region as a result of metallicity gradients. Thus, this extraplanar HII region (1) shows a slightly lower oxygen abundance than the inner disk.

Taking our results together with our previous work on NGC 55 (Tüllmann et al. 2003) and the results of other groups (see Sect. 1), it is clear that the mechanisms for forming extraplanar regions are diverse. The HII regions analyzed in this paper, along with most of the extraplanar HII regions mentioned in the literature, do not result from star formation in low-metallicity halo material. The extraplanar HII regions seem largely to result from material pulled out during an interaction or from ram pressure stripping. Therefore, the environment has a great impact on the generation of HII regions far above the disk plane.

We conclude that infalling gas, which is probably composed of falling back disk material and a small amount of intergalactic medium (e.g., Sancisi et al. 2008), does not form stars in the halo very frequently.

Acknowledgements. We thank the anonymous referee for constructive comments that helped improve this work. This research was supported in part by the DFG (German Research Foundation) research unit FOR1048, and has made use of IRAF, the NASA Astrophysics Data System Bibliographic Services, the GOLDMine Database, and the NASA/IPAC Extragalactic Database (NED), which is operated by the Jet Propulsion Laboratory, California Institute of Technology, under contract with the National Aeronautics and Space Administration.

\section{References}

Baldwin, J. A., Phillips, M. M., \& Terlevich, R. 1981, PASP, 93, 5

Barnes, J. E., \& Hernquist, L. 1996, ApJ, 471, 115

Cappellari, M. 2017, MNRAS, 466, 798

Cappellari, M., \& Emsellem, E. 2004, PASP, 116, 138

Cid Fernandes, R., Gu, Q., Melnick, J., et al. 2004, MNRAS, 355, 273

Comerón, F., Torra, J., Méndez, R. A., \& Gómez, A. E. 2001, A\&A, 366, 796

Cortese, L., Gavazzi, G., Boselli, A., \& Iglesias-Paramo, J. 2004, A\&A, 416, 119

de Vaucouleurs, G., de Vaucouleurs, A., Corwin, Jr., H. G., et al. 1991a, S\&T, 82,621

de Vaucouleurs, G., de Vaucouleurs, A., Corwin, Jr., H. G., et al. 1991b, Third Reference Catalogue of Bright Galaxies, Vol. I: Explanations and references, Vol. II: Data for galaxies between $0^{\mathrm{h}}$ and $12^{\mathrm{h}}$. Vol. III: Data for galaxies between $12^{\mathrm{h}}$ and $24^{\mathrm{h}}$

Engelbracht, C. W., Rieke, G. H., Gordon, K. D., et al. 2008, ApJ, 678, 804

Ferguson, A. M. N., Wyse, R. F. G., \& Gallagher, J. S. 1996, AJ, 112, 2567
Ferland, G. J., Korista, K. T., Verner, D. A., et al. 1998, PASP, 110, 761 Flores-Fajardo, N., Morisset, C., \& Binette, L. 2009, Rev. Mex. Astron. Astronfis., 45, 261

Garnett, D. R. 1992, AJ, 103, 1330

Garnett, D. R. 1998, in Abundance Profiles: Diagnostic Tools for Galaxy History, eds. D. Friedli, M. Edmunds, C. Robert, \& L. Drissen, ASP Conf. Ser., 147, 78

Gavazzi, G., Boselli, A., Donati, A., Franzetti, P., \& Scodeggio, M. 2003, A\&A, 400,451

Gerhard, O., Arnaboldi, M., Freeman, K. C., \& Okamura, S. 2002, ApJ, 580, L121

Greenstein, J. L., \& Sargent, A. I. 1974, ApJS, 28, 157

Gvaramadze, V. V., \& Bomans, D. J. 2008, A\&A, 490, 1071

Howarth, I. D. 1983, MNRAS, 203, 301

Howk, J. C., \& Savage, B. D. 1999, in The Physics and Chemistry of the Interstellar Medium, eds. V. Ossenkopf, J. Stutzki, \& G. Winnewisser, 38

Keenan, F. P., Lennon, D. J., Brown, P. J. F., \& Dufton, P. L. 1986, ApJ, 307, 694 Kenney, J. D. P., \& Koopmann, R. A. 1999, AJ, 117, 181

Kewley, L. J., \& Dopita, M. A. 2002, ApJS, 142, 35

Kewley, L. J., \& Ellison, S. L. 2008, ApJ, 681, 1183

Kewley, L. J., Dopita, M. A., Sutherland, R. S., Heisler, C. A., \& Trevena, J. 2001, ApJ, 556, 121

Kenney, J. D. P., van Gorkom, J. H., \& Vollmer, B. 2004, AJ, 127, 3361

Kilkenny, D., Luvhimbi, E., O’Donoghue, D., et al. 1995, MNRAS, 276, 906

Kobulnicky, H. A., \& Kewley, L. J. 2004, ApJ, 617, 240

Koopmann, R. A., Kenney, J. D. P., \& Young, J. 2001, ApJS, 135, 125

Kudritzki, R. P., Castro, N., Urbaneja, M. A., et al. 2016, ApJ, 829, 70

López-Sánchez, Á. R., Dopita, M. A., Kewley, L. J., et al. 2012, MNRAS, 426, 2630

Magrini, L., Bianchi, S., Corbelli, E., et al. 2011, A\&A, 535, A13

Mannucci, F., Cresci, G., Maiolino, R., et al. 2009, MNRAS, 398, 1915

Mendes de Oliveira, C., Cypriano, E. S., Sodré, L., \& Balkowski, C. 2004, in Outskirts of Galaxy Clusters: Intense Life in the Suburbs, ed. A. Diaferio, IAU Colloq., 195, 450

Modjaz, M., Kewley, L., Kirshner, R. P., et al. 2008, AJ, 135, 1136

Moustakas, J., Kennicutt, Jr., R. C., Tremonti, C. A., et al. 2010, ApJS, 190, 233

Nicholls, D. C., Dopita, M. A., \& Sutherland, R. S. 2012, ApJ, 752, 148

Oosterloo, T., \& van Gorkom, J. 2005, A\&A, 437, L19

Pettini, M., \& Pagel, B. E. J. 2004, MNRAS, 348, L59

Pilyugin, L. S. 2000, A\&A, 362, 325

Pilyugin, L. S. 2001, A\&A, 369, 594

Pilyugin, L. S., \& Thuan, T. X. 2005, ApJ, 631, 231

Pilyugin, L. S., Grebel, E. K., \& Kniazev, A. Y. 2014, AJ, 147, 131

Poveda, A., Ruiz, J., \& Allen, C. 1967, Boletin de los Observatorios Tonantzintla y Tacubaya, 4,86

Rossa, J., \& Dettmar, R. 2000, A\&A, 359, 433

Ryan-Weber, E. V., Putman, M. E., Freeman, K. C., Meurer, G. R., \& Webster, R. L. 2004, in Recycling Intergalactic and Interstellar Matter, eds. P.-A. Duc, J. Braine, \& E. Brinks, IAU Symp., 217, 492

Sánchez-Menguiano, L., Sánchez, S. F., Pérez, I., et al. 2016, A\&A, 587, A70

Sancisi, R., Fraternali, F., Oosterloo, T., \& van der Hulst, T. 2008, A\&ARv, 15, 189

Savage, B. D., \& Mathis, J. S. 1979, ARA\&A, 17, 73

Skillman, E. D., Bomans, D. J., \& Kobulnicky, H. A. 1997, ApJ, 474, 205

Slanger, T. G., Cosby, P. C., Osterbrock, D. E., Stone, R. P. S., \& Misch, A. A. 2003, PASP, 115, 869

Stasińska, G. 2002, ArXiv e-prints [arXiv: astro-ph/0207500]

Stasińska, G. 2010, in IAU Symp., 262, eds. G. Bruzual, \& S. Charlot, 93

Stone, R. C. 1982, ApJ, 261, 208

Tody, D. 1986, in Instrumentation in astronomy VI, ed. D. L. Crawford, Proc. SPIE, 627, 733

Tody, D. 1993, in Astronomical Data Analysis Software and Systems II, eds. R. J. Hanisch, R. J. V. Brissenden, \& J. Barnes, ASP Conf. Ser., 52, 173

Tüllmann, R., Rosa, M. R., Elwert, T., et al. 2003, A\&A, 412, 69

Tully, R. B. 1988, Nearby Galaxies Catalog (Cambridge University Press)

van Dokkum, P. G. 2001, PASP, 113, 1420

Vollmer, B. 2009, A\&A, 502, 427

Wilding, T., Alexander, P., \& Green, D. A. 1993, MNRAS, 263, 1075 